\title{
Balanced Cut Approximation in Random Geometric Graphs*
}

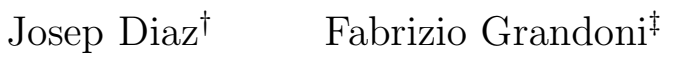 \\ Alberto Marchetti Spaccamela ${ }^{\S}$
}

\begin{abstract}
A random geometric graph $\mathcal{G}(n, r)$ is obtained by spreading $n$ points uniformly at random in a unit square, and by associating a vertex to each point and an edge to each pair of points at Euclidian distance at most $r$. Such graphs are extensively used to model wireless ad-hoc networks, and in particular sensor networks. It is well known that, over a critical value of $r$, the graph is connected with high probability.

In this paper we study the robustness of the connectivity of random geometric graphs in the supercritical phase, under deletion of edges. In particular, we show that, for a sufficiently large $r$, any cut which separates two components of $\Theta(n)$ vertices each contains $\Omega\left(n^{2} r^{3}\right)$ edges with high probability. We also present a simple algorithm that, again with high probability, computes one such cut of size $O\left(n^{2} r^{3}\right)$. From these two results we derive a constant expected approximation algorithm for the $\beta$-balanced cut problem on random geometric graphs: find an edge cut of minimum size whose two sides contain at least $\beta n$ vertices each.
\end{abstract}

Keywords: ad-hoc networks, sensor networks, random geometric graphs, balanced cut, approximation algorithms.

\section{Introduction}

Let us consider a wireless network of sensors on a terrain, where the sensors communicate by radio frequency, using an omnidirectional antenna. Each sensor broadcasts with the same power to the same distance. Two sensors can

\footnotetext{
*Corresponding author: Fabrizio Grandoni. A preliminary version of this paper appeared in ISAAC'06.

${ }^{\dagger}$ Departament de Llenguatges i Sistemes Informatics, Universitat Politecnica de Catalunya, Campus Nord - Ed. Omega, 240 Jordi Girona Salgado, 1-3 E-08034 Barcelona, diaz@lsi.upc.edu.

‡Dipartimento di Informatica, Sistemi e Produzione, Università di Roma "Tor Vergata", via del Politecnico 1, 00133 Roma, Italy, grandoni@disp.uniroma2.it.

$\S$ Dipartimento di Informatica e Sistemistica, "Sapienza" Università di Roma, via Ariosto 25, 00185, Roma, Italy, alberto.marchetti@dis.uniroma1.it.
} 
communicate if and only if they are within the transmission radius of each other. Sensor networks, and more in general ad-hoc wireless networks, are often modelled via random geometric graphs $[1,6]$. (For alternative models, see e.g. $[15,16]$ and references therein). A random geometric graph $\mathcal{G}(n, r)[10]$ is a graph resulting from placing a set $V$ of $n$ vertices uniformly at random on the unit square $[0,1]^{2}$, and connecting two vertices if and only if their Euclidean distance is at most the given radius $r$.

Random geometric graphs in general, and in particular their connectivity properties, have been intensively studied, both from the theoretical and from the empirical point of view. For the present paper, the most interesting result on random geometric graphs is the fact that, for $r=r(n)=\sqrt{(\ln n+c(n)) /(\pi n)}$, for any $c(n)$ such that $c(n) \rightarrow \infty$ when $n \rightarrow \infty, \mathcal{G}(n, r)$ is connected whp ${ }^{1}$ [17] (see also [12] for sharp connectivity thresholds). Once the connectivity is achieved, it is natural to wonder how robust it is: how many edges one needs to remove in order to disconnect the graph? In most applications the disconnection of one vertex, or of a few vertices, does not affect significantly the behavior of the network. So we can reformulate the question above in the following more general way: given $\beta \in[0,1 / 2]$, how many edges one needs to remove in order to isolate two components (not necessarily connected) of $\beta n$ vertices each?

Our results. We can formalize the question above in the following way. A cut of a graph is a partition of its vertices into two subsets $W$ and $B$, the sides of the cut. The size of cut $(W, B)$ is the number of edges $\delta(W, B)$ between $W$ and $B$. Given $\beta \in[0,1 / 2], \beta n \in \mathbb{N}$, a $\beta$-balanced cut is a cut where both sides contain at least $\beta n$ vertices. The $\beta$-balanced cut problem is to compute a $\beta$-balanced cut of minimum size. Here we prove that, if $r=r(n)=\sqrt{R \ln n / n}$ for $R \geq R^{*}$, with $R^{*}>1$ a sufficiently large constant, with high probability any $\beta$-balanced cut of $\mathcal{G}(n, r)$ has size $\Omega\left(\min \left\{\beta n R \log n, \sqrt{\beta n R^{3} \log ^{3} n}\right\}\right)$ (see Section 2).

We also present a simple algorithm that with high probability computes a cut of size $O\left(\min \left\{\beta n R \log n, \sqrt{\beta n R^{3} \log ^{3} n}\right\}\right)$, thus matching the lower bound (see Section 3 ). The two mentioned results imply a probabilistic constant approximation algorithm for the $\beta$-balanced cut problem. We eventually show how to extend such result to a constant expected approximation algorithm (see Section 3.1).

We observe that the minimum in the expressions above is given by the first term for $R>\beta n / \log n$, and by the second term for $R<\beta n / \log n$. With the threshold value $R=\beta n / \log n$, the expected number of neighbors of a node is $\Theta(\beta n)$.

We remark that the above results hold also if $R$ is a function of $n$, and that the hidden constants in the $O$ and $\Omega$ notations do not depend on $n, R$ and $\beta$.

Related Work. One of the first papers to study the $\beta$-balanced cut problem (for general graphs) is [3]. In this paper, the authors show that given a constant

\footnotetext{
${ }^{1}$ Throughout this paper, "whp" will abbreviate with high probability, that is with probability tending to 1 as $n$ goes to $\infty$.
} 
$\epsilon>0$, it is NP-hard to approximate the $\beta$-balanced cut problem within an additive term of $n^{2-\epsilon}$. The $\beta$-balanced cut problem admits a PTAS for $\beta \leq 1 / 3$, if the graph is dense, i.e. each vertex has degree $\Theta(n)$ [2]. For planar graphs there is a 2 -approximation for the $\beta$-balanced cut, if $\beta \leq 1 / 3$ [8].

For $\beta=1 / 2$, the $\beta$-balanced cut problem is the well-known minimum edge bisection problem. Minimum edge bisection is a difficult problem which has received a lot of attention due to its numerous applications (see e.g. [11]). It is known to be NP-Hard for general graphs [9], and in such case there is a $O\left(\log ^{1.5} n\right)$ approximation [7]. In the same paper, the authors prove that if the graph is planar, the approximation can be reduced to $O(\log n)$. In the case of random geometric graphs, it is known how to obtain a constant approximation to minimum edge bisection whp for the special case $R=R(n) \rightarrow \infty$ for $n \rightarrow \infty$ [5]. Our approximation algorithm improves on the algorithm in [5] in several ways: (i) it holds for arbitrary values of $\beta$ (not only for $\beta=1 / 2$ ), including the non-trivial special case that $\beta=o(1)$; (ii) it holds for constant values of $R$ as well; (iii) the value of the approximation ratio is constant in expectation, not only with high probability. These improvements are achieved by exploiting new and simpler techniques.

The deterministic counterpart of random geometric graphs are unit disk graphs [4]. An undirected graph is a unit disk graph if its vertices can be put in one-to-one correspondence with the centers of circles of equal radius in the plane in such a way that two vertices are joined by an edge if and only if the corresponding circles intersect. It is an open problem to prove the hardness of minimum edge bisection and $\beta$-balanced cut for unit disk graphs.

Preliminaries. Given a region $Q$ of the unit square, $|Q|$ denotes the area of $Q$, and $\|Q\|$ the number of points falling in $Q$. Note that $\|Q\|$ is a Binomial random variable of parameters $n$ and $|Q|$, for which the following standard Chernoff's Bounds hold [13]. Let $\mu=E[\|Q\|]=|Q| n$. Then:

$$
\begin{aligned}
& \operatorname{Pr}[\|Q\|<(1-\delta) \mu] \leq e^{-\delta^{2} \mu / 2} \quad \text { for } \delta \in[0,1) ; \\
& \operatorname{Pr}[\|Q\|>(1+\delta) \mu] \leq e^{-\delta^{2} \mu / 3} \quad \text { for } \delta \in[0,1) ; \\
& \operatorname{Pr}[\|Q\|>(1+\delta) \mu] \leq e^{-\delta^{2} \mu / 4} \quad \text { for } \delta \in[1,2 e-1) ; \\
& \operatorname{Pr}[\|Q\|>(1+\delta) \mu] \leq e^{-\delta \mu \ln 2} \quad \text { for } \delta \geq 2 e-1 \text {. }
\end{aligned}
$$

From now on $r=r(n)=\sqrt{R \ln n / n}$. For the sake of simplicity, we will assume $R=o(n / \log n)$. For $R=\Omega(n / \log n)$, the problems considered here become trivial. In particular, for $R \geq 2 n / \ln n$ the graph is a clique (deterministically). Moreover, we omit floors and ceilings when their role is not crucial. Without loss of generality, we assume that $n$ is lower bounded by a sufficiently large constant. 


\section{A Lower Bound}

In this section we show that, for any $\beta \in[0,1 / 2], \beta n \in \mathbb{N}$, and for $R \geq 240$, the size of any $\beta$-balanced cut for $\mathcal{G}(n, r)$ is $\Omega\left(\min \left\{\beta n R \log n, \sqrt{\beta n R^{3} \log ^{3} n}\right\}\right)$ with high probability.

In order to prove the mentioned lower bound, we consider a partition of the unit square into $5 n /(R \ln n)$ non-overlapping square cells of the same size. Each cell is adjacent to the cells to its right, left, top, and bottom. Observe that, since the side of each cell has length $L=\sqrt{R \ln n /(5 n)}$, a vertex is adjacent to all the vertices in the same cell and in all the adjacent cells: In fact, the maximum distance between two vertices in two adjacent cells (or in the same cell) is $\sqrt{5} L \leq \sqrt{R \ln n / n}=r$, where $r$ is the transmission radius. This property is crucial in the analysis. The number of points $\|C\|$ in each cell $C$ satisfies the following probabilistic bounds.

Lemma 1 For any $R \geq 240$, with probability $1-o\left(1 / n^{2}\right)$ all the cells $C$ of the partition above satisfy $R \ln n / 10 \leq\|C\| \leq 3 R \ln n / 10$.

Proof. Consider any cell $C$. Observe that $E[\|C\|]=R \ln n / 5$. By Chernoff's Bounds (1) and (3),

$\operatorname{Pr}\left(\|C\| \notin\left[\frac{R \ln n}{10}, \frac{3 R \ln n}{10}\right]\right) \leq e^{-(1 / 2)^{2} R \ln n / 10}+e^{-(1 / 2)^{2} R \ln n / 20}=O\left(1 / n^{3}\right)$.

The claim follows by applying the union bound to the $5 n /(R \ln n) \leq n /(48 \ln n)$ cells.

Let $(W, B)$ be any given cut, with $|W|=\beta n$. Let us call the vertices in $W$ white, and the vertices in $B$ black. A cell is white if at least one half of its points are white, otherwise the cell is black. We define a cluster $\mathcal{C}$ to be a maximal connected component of cells of the same color, with respect to the adjacency between cells defined above. The frontier $\partial \mathcal{C}$ of $\mathcal{C}$ is the subset of its cells which either touch the border of the unit square, or are adjacent to a cell of different color. We call good the cells of $\partial \mathcal{C}$ which are adjacent to a cell of different color, and bad the other cells of $\partial \mathcal{C}$. Observe that a cell is bad if it touches the border of the unit square and it is surrounded by cells of the same cluster (see Figure $1)$.

In order to prove the lower bound, we need the following two lemmas.

Lemma 2 Given a cluster of $k$ cells, its frontier contains at least $\sqrt{\pi k / 4}$ cells.

Proof. Suppose that the frontier contains $h<\sqrt{\pi k / 4}$ cells. Thus the perimeter of the cluster has length at most $4 h L$, where $L=\sqrt{R \ln n /(5 n)}$ is the length of the side of one cell. Such perimeter can enclose an area of size at most $(4 h L)^{2} /(4 \pi)$ (case of a disk of radius $4 h L /(2 \pi)$ ), and thus at most $4 h^{2} / \pi<k$ cells, which is a contradiction. 
Figure 1 Example of configuration of black and white cells. There are 3 black clusters and 1 white cluster.

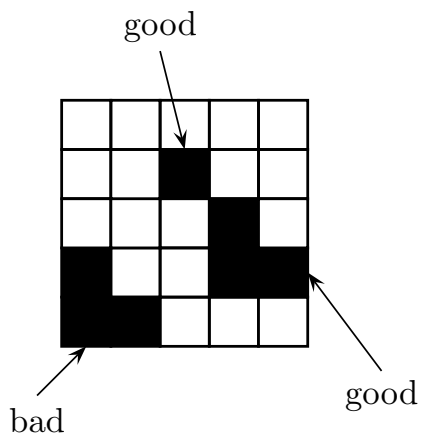

Lemma 3 Consider a cluster touching either 0 , or 1 , or 2 consecutive sides of the square. Then at least one third of the cells on its frontier are good.

Proof. Consider any cluster $\mathcal{C}$. Without loss of generality, let $\mathcal{C}$ be white. If $\mathcal{C}$ does not touch any side of the square, all the cells of $\partial \mathcal{C}$ are good. Thus the claim is trivially true.

Now suppose $\mathcal{C}$ touches one or two consecutive sides of the square, say the left side and possibly the top side. Let $\partial \mathcal{C}_{\text {good }}$ be the good cells of $\partial \mathcal{C}$, and $\partial \mathcal{C}_{\text {bad }}=\partial \mathcal{C} \backslash \partial \mathcal{C}_{\text {good }}$ the bad ones. Moreover, let $\partial \mathcal{C}^{\text {out }}$ be the cells of $\partial \mathcal{C}$ touching the border of the square, and $\partial \mathcal{C}^{i n}=\partial \mathcal{C} \backslash \partial \mathcal{C}^{\text {out }}$. Note that $\partial \mathcal{C}^{\text {in }} \subseteq \partial \mathcal{C}_{\text {good }}$ since the cells in $\partial \mathcal{C}^{\text {in }}$ do not touch any side of the square.

At least one half $\partial \mathcal{C}^{\prime}$ of the cells of $\partial \mathcal{C}^{\text {out }}$ touches one between the left and the top side of the square, say the left one. Consider any cell $C^{\prime} \in \partial \mathcal{C}^{\prime}$. If $C^{\prime}$ is bad, we can univocally associate to $C^{\prime}$ a good cell $C^{\prime \prime} \in \partial \mathcal{C}^{\text {in }}$ in the following way. Consider the sequence of consecutive white cells at the right of $C^{\prime}$ (there must be at least one such cell, since $C^{\prime}$ is bad). We let $C^{\prime \prime}$ be the rightmost of such cells. As a consequence, the number of good cells is lower bounded by $\left|\partial \mathcal{C}^{\prime}\right|$, and $\left|\partial \mathcal{C}_{\text {good }}\right| \geq\left|\partial \mathcal{C}^{\prime}\right| \geq\left|\partial \mathcal{C}^{\text {out }}\right| / 2$. Thus

$$
|\partial \mathcal{C}|=\left|\partial \mathcal{C}^{\text {in }}\right|+\left|\partial \mathcal{C}^{\text {out }}\right| \leq\left|\partial \mathcal{C}_{\text {good }}\right|+\left|\partial \mathcal{C}^{\text {out }}\right| \leq 3\left|\partial \mathcal{C}_{\text {good }}\right| .
$$

The claim follows.

We remark that Lemma 3 is asymptotically tight, as the example in Figure 2 shows.

Theorem 1 With probability $1-o\left(1 / n^{2}\right)$, for any $\beta \in[0,1 / 2], \beta n \in \mathbb{N}$, and for any $R \geq 240$, the size of any $\beta$-balanced cut of $\mathcal{G}(n, r)$ is

$$
\Omega\left(\min \left\{\beta n R \log n, \sqrt{\beta n R^{3} \log ^{3} n}\right\}\right) .
$$

Proof. By Lemma 1, with probability $1-o\left(1 / n^{2}\right)$ for each cell $C$,

$$
\|C\| \in\left[\frac{R \ln n}{10}, \frac{3 R \ln n}{10}\right] \text {. }
$$


Figure 2 For $h=4$, the white cluster $\mathcal{C}$ (touching two consecutive sides of the square) has $3 h-3$ cells on its frontier $\partial \mathcal{C}$, of which $h$ are good. Hence $\left|\partial \mathcal{C}_{\text {good }}\right| /|\partial \mathcal{C}|=1 / 3+1 /(3 h-3)$. The same example can be generalized to an arbitrarily large value of $h$.

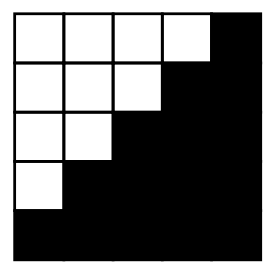

Thus it is sufficient to show that, given (5), the lower bound holds (deterministically) for any $\beta \in[0,1 / 2]$ and for any cut $(W, B)$ with $|W|=\beta n$.

We need some notation. By $\mathcal{W}$ and $\mathcal{B}$ we denote the set of white and black cells respectively. Moreover, $W_{\text {black }} \subseteq W$ ( $B_{\text {white }} \subseteq B$ ) is the subset of white (black) vertices in black (white) cells.

Since each vertex is adjacent to all the other vertices in the same cell, each vertex $w \in W_{\text {black }}$ contained into a (black) cell $C$ contributes with at least $\|C\| / 2 \geq R \ln n / 20$ edges to the edges of the cut. It follows that, if $\left|W_{\text {black }}\right| \geq$ $|W| / 2=\beta n / 2$, the size of the cut is at least

$$
\left|W_{\text {black }}\right| \frac{R \ln n}{20} \geq \frac{\beta n R \ln n}{40}=\Omega(\beta n R \log n) .
$$

Analogously, if $\left|B_{\text {white }}\right| \geq|B| / 2=(1-\beta) n / 2$, then the size of the cut is at least

$$
\left|B_{w h i t e}\right| \frac{R \ln n}{20} \geq \frac{(1-\beta) n R \ln n}{40}=\Omega(\beta n R \log n) .
$$

Thus, let us assume $\left|W_{\text {black }}\right|<|W| / 2$ and $\left|B_{\text {white }}\right|<|B| / 2$. Observe that, from this assumption and Equation (5),

$$
|\mathcal{W}| \geq \frac{\beta n / 2}{3 R \ln n / 10}=\frac{5 \beta n}{3 R \ln n} \quad \text { and } \quad|\mathcal{B}| \geq \frac{(1-\beta) n / 2}{3 R \ln n / 10}=\frac{5(1-\beta) n}{3 R \ln n}
$$

In particular, there are at least one black cell and one white cell.

By (5), since all the vertices in adjacent cells are adjacent, each pair of adjacent (good) cells $\left(C^{\prime}, C^{\prime \prime}\right)$, with $C^{\prime} \in \mathcal{W}$ and $C^{\prime \prime} \in \mathcal{B}$ contributes with at least

$$
\frac{\left\|C^{\prime}\right\|}{2} \frac{\left\|C^{\prime \prime}\right\|}{2} \geq \frac{R^{2} \ln ^{2} n}{400}=\Omega\left(R^{2} \log ^{2} n\right)
$$

distinct edges to the total number of edges in the cut. Since there must be at least one such pair $\left(C^{\prime}, C^{\prime \prime}\right)$, if $\beta=O(R \log n / n)$, trivially the size of the cut is $\Omega\left(R^{2} \log ^{2} n\right)=\Omega(\beta n R \log n)$. 
For $\beta=\Omega(R \log n / n)$ we need to bound the number of distinct pairs of type $\left(C^{\prime}, C^{\prime \prime}\right)$ in a more sophisticated way. In particular, we will show that the number of good cells, either white or black, is $\Omega(\sqrt{\beta n /(R \log n)})$, from which it follows that the size of the cut is at least

$$
\Omega\left(R^{2} \log ^{2} n\right) \Omega(\sqrt{\beta n /(R \log n)})=\Omega\left(\sqrt{\beta n R^{3} \log ^{3} n}\right) .
$$

We distinguish three sub-cases, depending on the existence of white clusters with some properties (see also Figure 3).

(i) There is a white cluster $\mathcal{C}$ touching either 3 or 2 opposite sides of the square (but not 4). Without loss of generality, let the right side of the square be untouched. Consider all the cells of $\mathcal{C}$ which have no cell of the same cluster to their right. Note that such cells belong to the frontier $\partial \mathcal{C}$ of the cluster. Moreover, they are all good (they have a black cell to their right). The number of such cells is $\sqrt{5 n /(R \ln n)}=\Omega(\sqrt{\beta n /(R \log n)})$.

(ii) Every white cluster touches 0,1 , or 2 consecutive sides of the square. Recall that the white cells are $|\mathcal{W}| \geq 5 \beta n /(3 R \ln n)$ by (6). Let $\mathcal{C}_{1}, \mathcal{C}_{2}, \ldots, \mathcal{C}_{p}$ be the $p$ white clusters. It follows by Lemmas 2 and 3 , that the total number of white good cells is at least

$$
\sum_{i=1}^{p} \frac{1}{3} \sqrt{\frac{\pi\left|\mathcal{C}_{i}\right|}{4}} \geq \frac{1}{3} \sqrt{\frac{\pi|\mathcal{W}|}{4}} \geq \frac{1}{3} \sqrt{\frac{\pi 5 \beta n}{12 R \ln n}}=\Omega(\sqrt{\beta n /(R \log n)}) .
$$

(iii) There is a white cluster touching the 4 sides of the square. It follows that each black cluster touches 0,1 , or 2 consecutive sides of the square. Thus, by basically the same argument as in case (ii), the number of black good cells is at least

$$
\frac{1}{3} \sqrt{\frac{\pi|\mathcal{B}|}{4}} \geq \frac{1}{3} \sqrt{\frac{\pi 5(1-\beta) n}{12 R \ln n}}=\Omega(\sqrt{\beta n /(R \log n)}) .
$$

This concludes the proof.

\section{A Simple Cutting Algorithm}

In this section we describe a simple algorithm simpleCut which, for a given input $\beta \in[0,1 / 2], \beta n \in \mathbb{N}$, computes a $\beta$-balanced cut. We will show that, for $R \geq 3 / \pi$, the size of the cut computed is $O\left(\min \left\{\beta n R \log n, \sqrt{\beta n R^{3} \log ^{3} n}\right\}\right)$ with high probability. This, together with Theorem 1, implies that simpleCut is a probabilistic constant approximation algorithm for the $\beta$-balanced cut problem for $R \geq 240$. We later show how to convert such result into a constant expected approximation algorithm. 
Figure 3 Example of cases (i), (ii) and (iii) in the proof of Theorem 1.

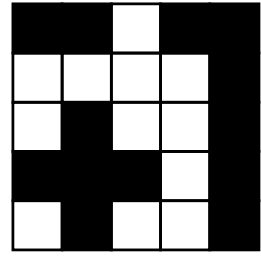

(i)

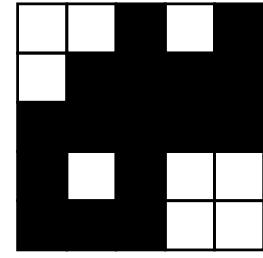

(ii)

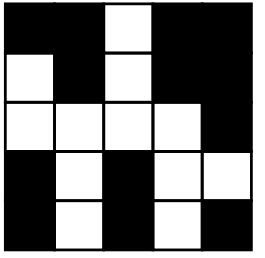

(iii)

Figure 4 The white disk $D$ contains one side $W$ of the cut, $\|W\|=\beta n$. The annulus $A$ of $D$, of width $\sqrt{R \ln n / n}$, is drawn in gray.

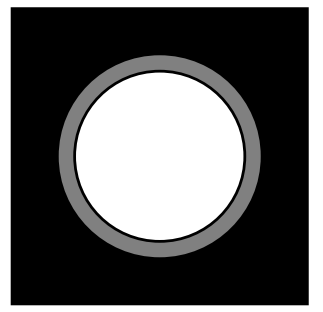

Algorithm 1 (simpleCut) Take the $\beta n$ vertices which are closest to $(1 / 2,1 / 2)$ (breaking ties arbitrarily). Such vertices form one side $W$ of the cut.

Observe that simpleCut can be easily implemented in polynomial time.

In order to bound the size of the cut produced by simpleCut, we need the following simple probabilistic bound on the degree of the vertices.

Lemma 4 For $R>3 / \pi$, the degree of all vertices of $\mathcal{G}(n, r)$ is upper bounded by $(3 \pi R \ln n)$ with probability $1-o\left(1 / n^{2}\right)$.

Proof. Consider the ball $B_{v}$ of radius $\sqrt{R \ln n / n}$ centered at a random vertex $v$. Clearly the degree of $v$ is $\left\|B_{v}\right\|-1$. We will show that $\operatorname{Pr}\left(\left\|B_{v}\right\|-1>\right.$ $3 \pi R \ln n)=o\left(1 / n^{3}\right)$. Then the lemma follows from the union bound. Observe that $B_{v}$ might not be entirely contained in the unit square (if $v$ is sufficiently close to the border). Let $B^{\prime}$ be a ball centered in $v$ such that its intersection with the unit square has area $\pi R \ln n / n$. Note that $B_{v} \subseteq B^{\prime}$. Moreover, $E\left[\left\|B^{\prime}\right\|\right]=$ $1+(n-1) \frac{\pi R \ln n}{n}=\pi R \ln n+1-o(1)$. By a simple coupling argument, and 
Chernoff's Bound (3),

$$
\begin{aligned}
\operatorname{Pr}\left(\left\|B_{v}\right\|-1>3 \pi R \ln n\right) & \leq \operatorname{Pr}\left(\left\|B^{\prime}\right\|-1>3 \pi R \ln n\right) \\
& \leq \operatorname{Pr}\left(\left\|B^{\prime}\right\|-1>3\left(E\left[\left\|B^{\prime}\right\|\right]-1\right)\right) \\
& \leq e^{-2^{2}\left(E\left[\left\|B^{\prime}\right\|\right]-1\right) / 4}=e^{-\pi R \ln n+o(1)}=o\left(1 / n^{3}\right) .
\end{aligned}
$$

Theorem 2 For any $\beta \in[0,1 / 2], \beta n \in \mathbb{N}$, and for $R>3 / \pi$, the size of the cut of $\mathcal{G}(n, r)$ computed by simpleCut is $O\left(\min \left\{\beta n R \log n, \sqrt{\beta n R^{3} \log ^{3} n}\right\}\right)$ with probability $1-o\left(1 / n^{2}\right)$.

Proof. Consider the cut $(W, B)$ computed by the algorithm, with $|W|=\beta n$. Trivially, the total degree of the edges incident to $W$ is an upper bound on the size of the cut. Hence, by Lemma 4 , with probability $1-o\left(1 / n^{2}\right)$ the size of the cut is at most:

$$
\beta n \cdot 3 \pi R \ln n=O(\beta n R \log n) .
$$

So, it is sufficient to show that, for $\beta=\Omega(R \log n / n)$, the size of the cut is $O\left(\sqrt{\beta n R^{3} \log ^{3} n}\right)$. In particular, $\beta \geq 8 \pi R \ln n / n$ is sufficient for our purposes.

Recall that, for a given region $Q$ of the unit square, $|Q|$ denotes the area of $Q$, and $\|Q\|$ the number of points inside $Q$. Let us denote by $D$ the disk centered in $(1 / 2,1 / 2)$, of minimum possible radius $\rho$, which contains all the vertices in $W$ (see Figure 4). In the following we will assume $\|D\|=\beta n$, i.e. there is exactly one vertex at distance $\rho$ from $(1 / 2,1 / 2)$ : this happens with probability one.

Let $A$ denote the annulus of width $\sqrt{R \ln n / n}$ surrounding $D$. The edges of the cut are a subset of the edges incident to the vertices in $A$ : In fact, any vertex outside $A \cup D$ is at distance larger than $\sqrt{R \ln n / n}$ from any vertex of $D$, and hence the corresponding edges cannot be part of the cut. Therefore, from Lemma 4 , it is sufficient to show that the number $\|A\|$ of vertices in $A$ is $O(\sqrt{\beta n R \log n})$ with probability $1-o\left(1 / n^{2}\right)$. From that we can conclude that the size of the cut is at most:

$$
3 \pi R \ln n \cdot O(\sqrt{\beta n R \log n})=O\left(\sqrt{\beta n R^{3} \log ^{3} n}\right) .
$$

Consider the disk $D^{\prime}$ centered in $(1 / 2,1 / 2)$ of radius $\rho^{\prime}=\sqrt{(3 / 2) \beta / \pi}$, and let $A^{\prime}$ be the annulus of width $\sqrt{R \ln n / n}$ surrounding $D^{\prime}$. Since $\rho^{\prime} \leq \sqrt{3 /(4 \pi)}<1 / 2$, for $n$ large enough $D^{\prime}$ and $A^{\prime}$ are entirely contained in the unit square.

Observe that, given $\rho \leq \rho^{\prime}$, the density of points in both $A$ and $A^{\prime}$ is the same, that is $(n-\beta n) /(1-|D|)$. Under the same assumption, this density is maximized when $\rho=\rho^{\prime}$ (and hence $|D|$ is the largest possible). Thus, for any $c>0$,

$$
\operatorname{Pr}\left[\|A\|>c \mid \rho \leq \rho^{\prime}\right] \leq \operatorname{Pr}\left[\left\|A^{\prime}\right\|>c \mid \rho \leq \rho^{\prime}\right] \leq \operatorname{Pr}\left[\left\|A^{\prime}\right\|>c \mid \rho=\rho^{\prime}\right] .
$$


We remark that the upper bound above holds even if the event $\rho=\rho^{\prime}$ happens with probability zero. For $\rho=\rho^{\prime},|D|=\pi(3 / 2) \beta / \pi$ and hence $\frac{n-\beta n}{1-|D|}=\frac{n-\beta n}{1-3 \beta / 2}$. We also observe that

$$
\left|A^{\prime}\right|=\pi\left(\sqrt{\frac{3 \beta}{2 \pi}}+\sqrt{\frac{R \ln n}{n}}\right)^{2}-\pi\left(\sqrt{\frac{3 \beta}{2 \pi}}\right)^{2}=\pi \sqrt{\frac{R \ln n}{n}}\left(2 \sqrt{\frac{3 \beta}{2 \pi}}+\sqrt{\frac{R \ln n}{n}}\right) .
$$

Therefore, multiplying the density of points in $A^{\prime}$ by its area,

$$
\mu:=E\left[\left\|A^{\prime}\right\| \mid \rho=\rho^{\prime}\right]=\frac{n-\beta n}{1-3 \beta / 2} \pi \sqrt{\frac{R \ln n}{n}}\left(2 \sqrt{\frac{3 \beta}{2 \pi}}+\sqrt{\frac{R \ln n}{n}}\right) .
$$

In particular

$$
\sqrt{108} \ln n \leq \sqrt{(3 / 2) \pi \beta R n \ln n} \leq \mu \leq 12 \sqrt{(3 / 2) \pi \beta R n \ln n} .
$$

It follows from Chernoff's Bound (3) that

$$
\operatorname{Pr}\left[\left\|A^{\prime}\right\|>2 \mu \mid \rho=\rho^{\prime}\right] \leq e^{-\mu / 4} \leq e^{-\sqrt{108} \ln n / 4}=o\left(1 / n^{2}\right) .
$$

Moreover, being $E\left[\left\|D^{\prime}\right\|\right]=(3 / 2) \beta n$, from Chernoff's Bound (1),

$$
\operatorname{Pr}\left[\rho>\rho^{\prime}\right]=\operatorname{Pr}\left[\left\|D^{\prime}\right\|<\beta n\right] \leq e^{-(1 / 3)^{2}(3 / 2) \beta n / 2} \leq e^{-\beta n / 12}=o\left(1 / n^{2}\right) .
$$

Altogether

$$
\begin{aligned}
\operatorname{Pr}[\|A\|>2 \mu] & \leq \operatorname{Pr}\left[\rho>\rho^{\prime}\right]+\operatorname{Pr}\left[\|A\|>2 \mu \mid \rho \leq \rho^{\prime}\right] \operatorname{Pr}\left[\rho \leq \rho^{\prime}\right] \\
& \leq o\left(1 / n^{2}\right)+\operatorname{Pr}\left[\left\|A^{\prime}\right\|>2 \mu \mid \rho=\rho^{\prime}\right] \\
& =o\left(1 / n^{2}\right) .
\end{aligned}
$$

It follows that $\|A\| \leq 2 \mu=O(\sqrt{\beta n R \log n})$ with probability $1-o\left(1 / n^{2}\right)$.

Corollary 1 For any $\beta \in[0,1 / 2], \beta n \in \mathbb{N}$, and for any $R \geq 240$, with probability $1-o\left(1 / n^{2}\right)$ Algorithm simpleCut computes a constant approximation for the $\beta$-balanced cut problem on $\mathcal{G}(n, r)$.

Proof. It follows immediately from Theorems 1 and 2.

\subsection{From Probabilistic to Expected Approximation}

We have shown that Algorithm simpleCut is a probabilistic constant approximation algorithm for the $\beta$-balanced cut problem. We next show how to extend this result to a constant expected approximation algorithm for the same problem.

Under the assumption that the optimal cut has size at least one, Algorithm simpleCut has the desired properties. In fact, in that case, any cut (including 
the one returned by simpleCut), is a $O\left(n^{2}\right)$ approximation of the optimum in the worst case. Hence, the unlikely event that one of the bounds given by Theorems 1 and 2 does not hold contributes only with a term $o(1)$ to the approximation factor of simpleCut. However, this argument does not work when the optimal cut has size zero (event which happens with positive probability).

For this reason, we introduce the following algorithm zeroCut to compute a cut of size zero, if any. Compute the connected components of $\mathcal{G}(n, r)$. For any integer $m, \beta n \leq m \leq n / 2$, check whether there is a subset of components whose total size is $m$. If yes, return such subset of components as one side of the partition. Note that for each of the $O(n)$ possible values of $m$, we have to solve an instance of the subset sum problem (where the sizes of the components form the input list, and the target value is $m$ ). The subset sum problem is NP-hard in general, but it can be solved in polynomial time via dynamic programming when the input values are polynomially-bounded integers [9]. This is the case here (all the sizes are integers between 1 and $n$ ). Combining zeroCut and simpleCut, one obtains the desired constant expected approximation algorithm.

Algorithm 2 (refinedCut) If zeroCut returns a solution, return it. Otherwise, return the solution computed by simpleCut.

Theorem 3 For any $\beta \in[0,1 / 2], \beta n \in \mathbb{N}$, and for any $R \geq 240$, refinedCut is a constant expected approximation algorithm for the $\beta$-balanced cut problem on $\mathcal{G}(n, r)$.

Proof. Let $z^{H}$ and $z^{*}$ denote the size of the solution found by refinedCut and the size of the optimum cut, respectively. Let moreover $\mathcal{A}$ denote the event that

$$
z^{*} \geq c \min \left\{\beta n R \log n, \sqrt{\beta n R^{3} \log n^{3}}\right\}
$$

and

$$
z^{H} \leq C \min \left\{\beta n R \log n, \sqrt{\beta n R^{3} \log n^{3}}\right\},
$$

where the constants $c$ and $C$ are as in the proofs of Theorems 1 and 2. Note that $\operatorname{Pr}[\mathcal{A}]=1-o\left(1 / n^{2}\right)$. Given $\mathcal{A}$, the approximation ratio of refinedCut is at most $C / c=O(1)$. Given $\overline{\mathcal{A}}$, if the size of the optimum cut is zero, zeroCut computes the optimum solution and the approximation ratio is 1 by definition. Otherwise, any cut, and hence also the cut computed by simpleCut, is a $O\left(n^{2}\right)$ approximation. Altogether the expected approximation ratio is

$$
E\left(z^{H} / z^{*}\right)=\operatorname{Pr}[\mathcal{A}] O(1)+\operatorname{Pr}[\overline{\mathcal{A}}] O\left(n^{2}\right)=O(1) .
$$

Remark 1 The threshold 240 can be reduced to a value arbitrarily close to 30 by adapting the constants in Lemma 1. However, this would increase the approximation ratio. If we only desire a probabilistic constant approximation, such threshold can be made arbitrarily close to 10, with the same drawback as above. 
Acknowledgments. This work was partially supported by EU Integrated Project AEOLUS (FET-15964). The first author was partially supported by La distinció de la Generalitat de Catalunya, 2002, the second author by project MAINSTREAM of the Italian Ministry of University and Research and the third author by EU STREP Project ICT-215270 FRONTS and by FIRB project RBIN047MH of the Italian Ministry of University and Research.

\section{References}

[1] I. Akyildiz, W. Su, Y. Sankarasubramaniam, and E. Cayirci. Wireless sensor networks: a survey. Computer Networks, 38:393-422, 2002.

[2] S. Arora, D. Karger, and M. Karpinski. Polynomial time approximation schemes for dense instances of NP-hard problems. In ACM Symposium on the Theory of Computing (STOC), pages 284-293, 1995.

[3] T.N. Bui, and C. Jones. Finding good approximate vertex and edge partitions is NP-hard. Information Processing Letters, 42:153-159, 1992.

[4] N.B. Clark, C.J. Colbourn, and D.S. Johnson. Unit disk graphs, Discrete Mathematics, 86:165-177, 1990.

[5] J. Díaz, M. Penrose, J. Petit, and M. Serna. Approximating layout problems on random geometric graphs. Journal of Algorithms, 39:78-116, 2001.

[6] J. Díaz, J. Petit, and M. Serna. Evaluation of basic protocols for optical smart dust networks. IEEE Transactions on Mobile Networks, 2:189-196, 2003.

[7] U. Feige, and R. Krauthgamer. A polylogarithmic approximation of the minimum bisection. SIAM Journal on Computing, 31(3): 1090-1119, 2002.

[8] G. Garg, H. Saran, and V. Vazirani. Finding separator cuts in planar graphs within twice the optimal. In IEEE Symposium on Foundations of Computer Science (FOCS), pages 14-23, 1994.

[9] M. Garey, and D. Johnson. Computers and Intractability. Freeman. N.Y., 1979.

[10] E. Gilbert. Random plane networks. Journal of the Society for Industrial and Applied Mathematics, 9:533-543, 1961.

[11] S.H. Gerez. Algorithms for VLSI design automation. Wiley, 2003.

[12] A. Goel, S. Rai, and V. Krishnamachari. Sharp thresholds for monotone properties in random geometric graphs. In ACM Symposium on Foundations of Computer Science (FOCS), pages 13-23, 2004.

[13] R. Motwani and P. Raghavan. Randomized Algorithms. Cambridge University Press, 1995.

[14] S. Muthukrishnan and G. Pandurangan. The Bin-covering technique for thresholding random geometric graph properties graphs. In ACM-SIAM Symposium on Discrete Algorithms (SODA), pages 989-998, 2005.

[15] S. E. Nikoletseas, C. Raptopoulos, and P. G. Spirakis. Large independent sets in general random intersection graphs. Theoretical Computer Science, 406(3):215$224,2008$.

[16] S. E. Nikoletseas and P. G. Spirakis. Efficient sensor network design for continuous monitoring of moving objects. Theoretical Computer Science, 402(1):56-66, 2008. 
[17] M. Penrose. Random Geometric Graphs. Oxford Studies in Probability. Oxford U.P., 2003. 